\title{
Derivation and analysis of two-dimensional composition space equations for multi-regime combustion using orthogonal coordinates
}

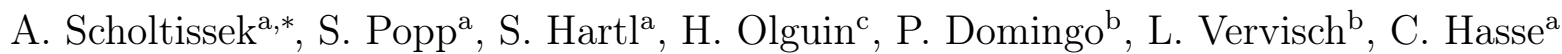 \\ ${ }^{a}$ Institute for Simulation of reactive Thermo-Fluid Systems, TU Darmstadt, Otto-Berndt-Straße 2, 64287 \\ Darmstadt, Germany \\ ${ }^{b}$ CORIA - CNRS, Normandie Université, INSA de Rouen, Technopôle du Madrillet, BP 8, 76801 \\ Saint-Étienne-du-Rouvray, France \\ ${ }^{c}$ Department of Mechanical Engineering, Universidad Técnica Federico Santa María, Avenida España 1680, \\ Valparaíso, Chile
}

\begin{abstract}
Interactions between premixed and non-premixed reaction zones can lead to complex mixed combustion regimes, here denoted as multi-regime combustion, which pose challenges to many conventional combustion modeling approaches. Such conditions occur in most practical combustors and can originate from partial premixing, mixture inhomogeneities/stratification, hot product recirculation, or local flame extinction and re-ignition. Therefore, novel equations are derived for modeling multi-regime combustion which are formulated with respect to a twodimensional composition space spanned by mixture fraction and reaction progress variable. Contrary to previous works, the dependency of the progress variable on the mixture fraction is considered in the new model. This is achieved by splitting the progress variable gradient into an aligned and an orthogonal component with respect to the mixture fraction gradient and the latter is used to define the second coordinate. In the theory that follows, a balance equation for the progress variable on mixture fraction iso-surfaces is formulated. Using this balance equation together with the orthogonal coordinate system, the transformation of species and temperature equations to the 2D composition space yields a novel set of equation without so-called cross-terms. This is advantageous since cross-terms obtained with previous approaches lack a general closure and it is uncertain if it exists at all. Furthermore, the approach allows to naturally distinguish between non-premixed and premixed combustion regimes, auto ignition, and it covers multi-regime combustion characteristics. The theory is validated and discussed by means of a fully resolved solution of a laminar triple flame using detailed chemistry. At first, regions which exhibit premixed, non-premixed or multi-regime combustion characteristics are identified. The triple flame solution then serves as a database from which all relevant theoretical relations are post-processed and validated. In comparison to budgets of conventional 1D flamelet equations for premixed and non-premixed combustion it is shown that only the full set of transport terms considered in the 2D equations accurately balances chemical source terms everywhere in the triple flame, especially in regions where
\end{abstract}


multi-regime combustion prevails.

\section{Introduction}

The distinction between the premixed and non-premixed combustion regime has been essential in the development of combustion theory and modeling. However, practical combustion systems often exhibit, sometimes by design, complex flame structures which cannot be characterized as purely premixed or purely non-premixed [1-4]. Instead, effects such as partial premixing, mixture inhomogeneities/stratification, hot product recirculation, entrainment of oxidizer, and local flame extinction can lead to mixed combustion regimes which are denoted as multi-regime combustion in this work. For the last decade, multi-regime combustion has been a topical research area resulting in a broad range of experimental and numerical works as reviewed by Masri [2. To date, modeling of multi-regime combustion still poses a major challenge since many combustion models employed in CFD simulations of practical combustors rely on restrictive assumptions regarding the structure of reaction zones.

The identification of combustion regimes with respect to premixed, non-premixed or multiregime characteristics is non-trivial and multiple works exist regarding this topic. Yamashita et al. [5] suggested the flame index, a scalar which considers the alignment of fuel and oxidizer gradients. The flame index characterizes a flame as premixed if the scalar product of the local fuel and oxidizer gradient is positive and as non-premixed if it is negative. This criterion was later used for analyzing well-resolved numerical data and also extended by other groups [6] 10. Another combustion regime indicator was proposed by Knudsen and Pitsch [1] who assessed flame structures based on a balance of the local chemical source term, molecular mixing, and unsteady effects. In comparison to the flame index, their combustion regime index showed comparable predictions, but differences were found for regions where premixed and non-premixed flame structures occur in close proximity. While the aforementioned combustion regime identifiers rely on scalar gradient information, which is only accessible from well-resolved numerical simulations, Hartl et al. [12] developed a gradient free regime identification (GFRI) approach. The method can also be applied to experimental data and allows distinguishing combustion regimes based on the local thermochemical state obtained from Raman/Rayleigh spectroscopy. Since, the GFRI approach has been refined to identify the relative importance of combustion regimes [3] and to differentiate local combustion regime characteristics [4].

Besides the analytical tools for combustion regime identification, different modeling approaches exist to describe multi-regime combustion characteristics. Here, a composition space modeling approach is used and related previous works are briefly revisited in the following. Well-established composition space models, such as the classical non-premixed

\footnotetext{
*Corresponding author:

Email address: scholtissek@stfs.tu-darmstadt.de (A. Scholtissek)
} 
flamelet model [13], Flamelet Generated Manifolds (FGM) [14] and Flame Prolongation of ILDM (FPI) 15] rely on a one-dimensional representation of the reaction layer (non-premixed or premixed, respectively). It has been shown that these modeling approaches can capture multi-regime/partially premixed combustion characteristics to a certain extent [6, 8, 10, 14, 16 19. However, as stated initially, these models rely on restrictive a-priori assumptions and can exhibit substantial modeling errors for multi-regime combustion characteristics. A computationally inexpensive, albeit non-generic, approach to remedy these shortcomings is the blending between premixed and non-premixed 1D flamelet solutions, e.g. based on some sort of modified flame index [6, 10, 11, 20]. While this approach naturally recovers the limiting cases of premixed and non-premixed combustion, it ignores complex interactions between premixed and non-premixed reaction zones (i.e. multi-regime characteristics) which are essential for the accurate prediction of certain flame structures [2 4].

A first attempt to describe this sort of interaction in a two-dimensional composition space was published by Domingo et al. [21] who derived flamelet-like equations with respect to the conditioning variables mixture fraction and progress variable. They showed that the modeling approach can distinguish between auto ignition, premixed, partially-premixed, and non-premixed combustion based on a series of Damköhler numbers. The approach was extended and generalized in the subsequent work by Nguyen et al. 22] further including the numerical solution of 2D composition space equations. A slightly different approach was presented by Knudsen and Pitsch [1, 11] who derived 2D composition space equations with respect to the mixture fraction and the reaction progress parameter $\Lambda$, a scalar quantity which is set to the value of progress variable at stoichiometry and held fixed across iso-mixture fraction surfaces. Using the progress-variable-like scalar $\Lambda$ as a conditioning variable instead of the progress variable itself has the advantage that it is statistically independent from the mixture fraction. Nevertheless, other difficulties arise: it is unfeasible to transport $\Lambda$ in a CFD context since its transport equation is too complex and it is not straight-forward to recover the premixed and non-premixed mode of combustion from the equations [11]. For the sake of completeness other two-dimensional composition space models are mentioned here: equation sets were published utilizing two mixture fractions as conditioning variables to model multiple fuel injections in Diesel engines [23, 24], and utilizing mixture fraction and enthalpy to capture mixing and thermal stratification in HCCI engines [25, 26]. Similar approaches have also been presented for Conditional Moment Closure (CMC) [27].

Despite the more advanced representation of reaction zones, 2D composition space modeling has not been widely adopted, yet. This has several reasons: first, 2D composition space models rely on closure strategies for the scalar dissipation rates (or gradients) of the conditioning variables and the so-called cross-terms which additionally appear in the equations. Only recently it has been shown that a closure for the progress variable gradient (or its scalar dissipation rate) requires an additional equation to be solved [28, 29], since no general model exists for this quantity. Second, while the classical flamelet equations are simple to solve and implement, 2D composition space models are significantly more complex. Third, the aspect of the statistical independence of the conditioning variables, as addressed 
by Knudsen and Pitsch [1, 11, is not always rigorously incorporated in the composition space transformation.

The main motivation of this work is to advance the multi-dimensional composition space theory to overcome the aforementioned shortcomings. More specifically, the objective of this paper is twofold: (1) develop a consistent theory in a two-dimensional composition space to describe multi-regime combustion (c.f. Sec. 2) and (2) analyze and validate the approach in terms of a well-known laminar triple flame setup (c.f. Sec. 3). Thereby, this work shall provide a conceptual basis for modeling multi-regime combustion in CFD simulations with tabulated chemistry methods.

\section{General formulation}

In this section composition space equations for termochemical scalars are derived and discussed. The derivation consists of three steps: in Sec. 2.1, the evolution of thermochemical quantities is expressed in terms of mixture fraction isosurfaces serving as a Lagrangian frame of reference. Thereafter, an orthogonal coordinate system is introduced and used to project thermochemical scalars into composition space (Sec. 2.2). The composition space equations are presented and examined in Sec. 2.3 .

It is then demonstrated how the composition space equations recover the limiting cases of combustion (Sec. 2.4), which is followed by a discussion of conceivable closures, cross-terms and differential diffusion (Sec. 2.5).

\subsection{Lagrangian reference frame}

Let us introduce the mixture fraction $Z(\mathbf{x}, t)$, a passive scalar $(Z=1$ in fuel feeding stream and $Z=0$ in oxidiser stream), whose balance equation reads (unity Schmidt and Lewis numbers) [30]

$$
\rho \frac{\partial Z}{\partial t}+\rho \mathbf{u} \cdot \nabla Z=\nabla \cdot(\rho D \nabla Z)
$$

where $\rho$ is the density, $\mathbf{u}$ is the velocity vector and $D$ is the diffusion coefficient. The motion of an arbitrary $Z$-isosurface is described by the kinematic condition [31]

$$
\frac{\mathrm{D} Z}{\mathrm{D} t}=\frac{\partial Z}{\partial t}+\mathbf{u}_{p} \cdot \nabla Z=0
$$

where $\mathbf{u}_{p}$ can be interpreted as the velocity of a mass-less particle $p$ attached to the $Z$-isosurface. Using Eq. (2) to replace the transient term in Eq. (1) yields [32]

$$
\mathbf{u}_{p}=\mathbf{u}-\left[\frac{1}{\rho|\nabla Z|} \nabla \cdot(\rho D \nabla Z)\right] \mathbf{n}
$$

where the unit vector $\mathbf{n}=\nabla Z /|\nabla Z|$ is the normalized mixture fraction gradient. 
Let us now consider the chemical species $Y_{i}(\mathbf{x}, t)$ (analogously other thermochemical scalars) verifying a balance equation of the usual form (unity Lewis numbers)

$$
\rho \frac{\partial Y_{i}}{\partial t}+\rho \mathbf{u} \cdot \nabla Y_{i}=\nabla \cdot\left(\rho D \nabla Y_{i}\right)+\dot{\omega}_{i}
$$

where $\dot{\omega}_{i}$ is the chemical source. The evolution of the the species $Y_{i}$ at the particle position is defined by the material derivative

$$
\frac{\mathrm{D} Y_{i}}{\mathrm{D} t}=\frac{\partial Y_{i}}{\partial \tau}=\frac{\partial Y_{i}}{\partial t}+\mathbf{u}_{p} \cdot \nabla Y_{i}
$$

where $\tau$ is a time-like variable which is independent from the coordinate variables attached to the particle $p$ (i.e. on a $Z$-isosurface). Using Eq. (4) to replace the transient term $\partial Y_{i} / \partial t$ we can rewrite Eq. (5)

$$
\frac{\partial Y_{i}}{\partial \tau}=\frac{1}{\rho} \nabla \cdot\left(\rho D \nabla Y_{i}\right)+\frac{\dot{\omega}_{i}}{\rho}-\frac{1}{\rho} \nabla \cdot(\rho D \nabla Z) \frac{\partial Y_{i}}{\partial Z}
$$

where the partial derivative of $Y_{i}$ with respect to $Z$ is given by

$$
\frac{\partial Y_{i}}{\partial Z}=\frac{1}{|\nabla Z|} \mathbf{n} \cdot \nabla Y_{i}=\frac{\nabla Z \cdot \nabla Y_{i}}{|\nabla Z|^{2}}
$$

Equation 6 represents a species balance equation valid for any $Z$-isosurface and forms the basis for the following derivation. In case important physics occur along the normal $\mathbf{n}$ only, i.e. as for a thin non-premixed flame, a one-dimensional structure can be assumed and the composition space transformation of Eq. 6] leads to the unsteady flamelet equations [13]. For multi-regime combustion however, at least a second conditioning variable is required, which is considered next.

\subsection{Orthogonal scalar coordinates}

An orthogonal composition-space system of coordinates $\left(\mathbf{n}_{1}, \mathbf{n}_{2}, \mathbf{n}_{3}\right)$ may be constructed from $Z$ and any reactive scalar $Y_{c}(\mathbf{x}, t)$ described by

$$
\rho \frac{\partial Y_{c}}{\partial t}+\rho \mathbf{u} \cdot \nabla Y_{c}=\nabla \cdot\left(\rho D \nabla Y_{c}\right)+\dot{\omega}_{c}
$$




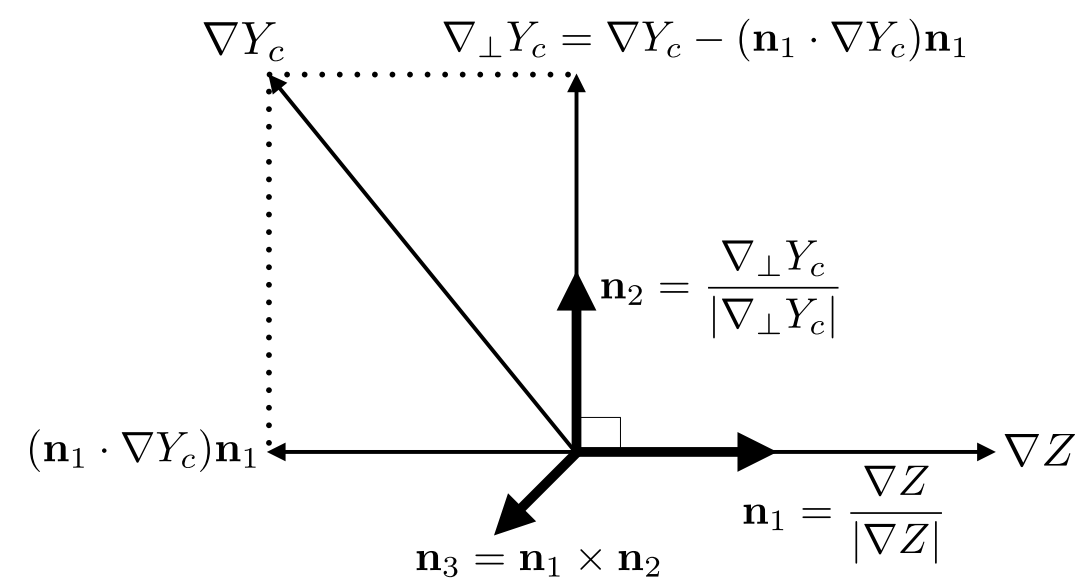

Figure 1: Sketch of the orthogonal scalar coordinate system in the physical space.

using the corresponding vector coordinates (Fig. 1)

$$
\begin{aligned}
& \mathbf{n}_{1}=\frac{\nabla Z}{|\nabla Z|}, \\
& \mathbf{n}_{2}=\frac{\nabla Y_{c}-\left(\mathbf{n}_{1} \cdot \nabla Y_{c}\right) \mathbf{n}_{1}}{\left|\nabla Y_{c}-\left(\mathbf{n}_{1} \cdot \nabla Y_{c}\right) \mathbf{n}_{1}\right|}=\frac{\nabla_{\perp} Y_{c}}{\left|\nabla_{\perp} Y_{c}\right|}, \\
& \mathbf{n}_{3}=\mathbf{n}_{1} \times \mathbf{n}_{2}=\frac{\nabla e}{|\nabla e|} .
\end{aligned}
$$

Here, the reactive scalar $Y_{c}$ is defined as a weighted sum of species

$$
Y_{c}=\sum_{i}^{n_{s}} \alpha_{i} Y_{i}
$$

a common definition in the literature [14, 33, 34] and it will be denoted as reaction progress variable in the following. The gradient

$$
\nabla_{\perp} Y_{c}=\nabla Y_{c}-\left(\mathbf{n}_{1} \cdot \nabla Y_{c}\right) \mathbf{n}_{1} \stackrel{(7)}{=} \nabla Y_{c}-\frac{\partial Y_{c}}{\partial Z} \nabla Z
$$

is the orthogonal component of $\nabla Y_{c}$ with respect to $\nabla Z$ and $e$ is a coordinate along of which changes of thermochemical quantities are assumed small (similar as in [11]). The definition of the second coordinate, Eq. (10), ensures orthogonality, i.e. $\mathbf{n}_{1} \cdot \mathbf{n}_{2}=0$. In this $\left(\mathbf{n}_{1}, \mathbf{n}_{2}, \mathbf{n}_{3}\right)$ 

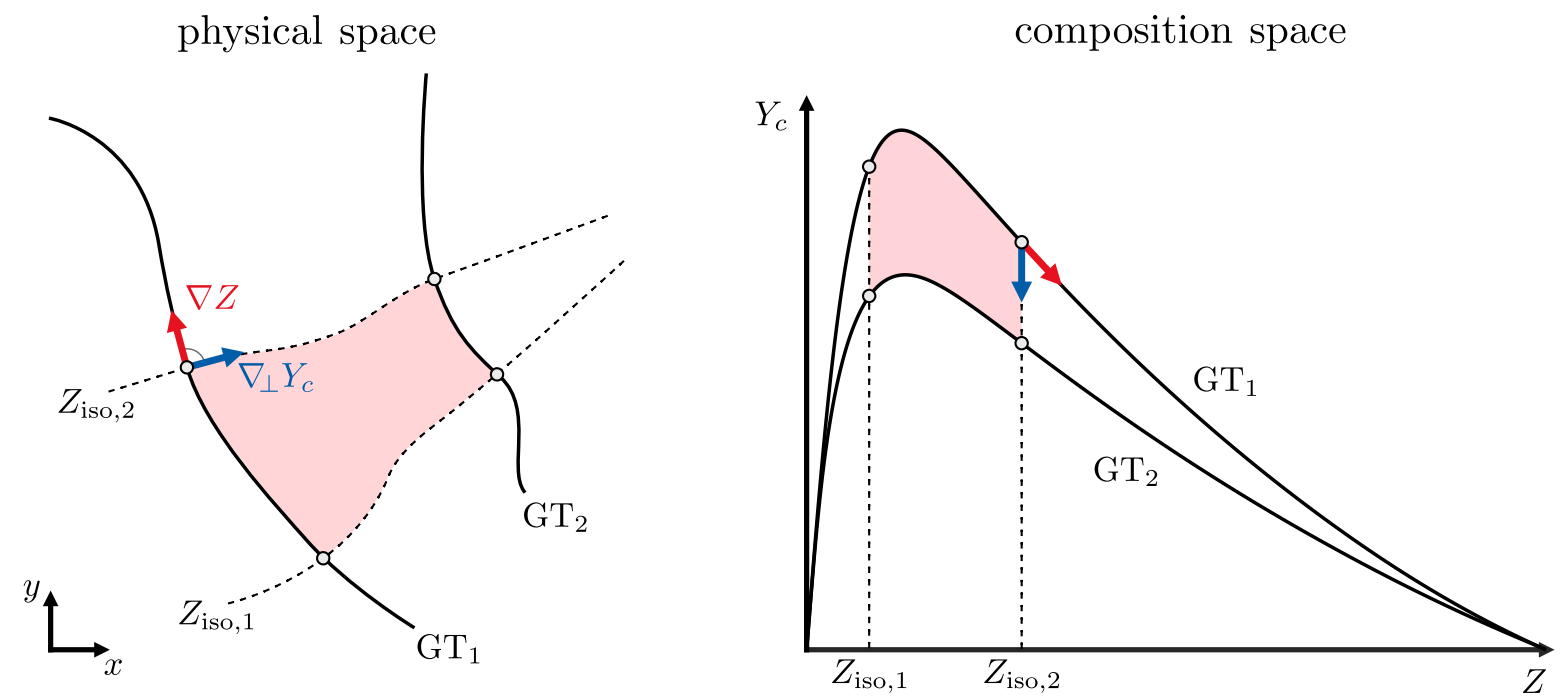

Figure 2: In the physical space (left), two mixture fraction gradient trajectories (GT) and two $Z$-isolines intersect at four points. The same points are mapped into the composition space parametrized by $Z$ and $Y_{c}$ (right). Traversing points along the gradient trajectories and $Z$-isolines in the physical space leads to a corresponding motion in composition space in the direction of the red and blue arrows, respectively.

orthogonal coordinate system, usual projection rules apply and $\nabla Y_{i}$ reads

$$
\begin{aligned}
\nabla Y_{i} & =\left(\mathbf{n}_{1} \cdot \nabla Y_{i}\right) \mathbf{n}_{1}+\left(\mathbf{n}_{2} \cdot \nabla Y_{i}\right) \mathbf{n}_{2}+\left(\mathbf{n}_{3} \cdot \nabla Y_{i}\right) \mathbf{n}_{3} \\
& =\frac{\partial Y_{i}}{\partial Z}|\nabla Z| \mathbf{n}_{1}+\left.\frac{\partial Y_{i}}{\partial Y_{c}}\right|_{Z}\left|\nabla_{\perp} Y_{c}\right| \mathbf{n}_{2}+\frac{\partial Y_{i}}{\partial e}|\nabla e| \mathbf{n}_{3} \\
& =\frac{\partial Y_{i}}{\partial Z} \nabla Z+\left.\frac{\partial Y_{i}}{\partial Y_{c}}\right|_{Z} \nabla_{\perp} Y_{c}+\frac{\partial Y_{i}}{\partial e} \nabla e
\end{aligned}
$$

where the definition

$$
\left.\frac{\partial Y_{i}}{\partial Y_{c}}\right|_{Z}=\frac{\nabla Y_{i} \cdot \nabla_{\perp} Y_{c}}{\left|\nabla_{\perp} Y_{c}\right|^{2}},
$$

is used to represent the derivative of the thermochemical quantity $Y_{i}$ projected into composition space along $\nabla_{\perp} Y_{c}$. Note that this derivative of $Y_{i}$ with respect to $Y_{c}$ is defined for constant mixture fractions, which is indicated by the notation $\left.(\cdot)\right|_{Z}$ and directly follows from the construction of the orthogonal coordinate system (c.f. Fig. 1). As previously stated, derivatives of thermochemical variables with respect to $e$ are assumed small and are therefore omitted in the following.

Before further usage, it is useful to examine the mapping from physical to composition space by the orthogonal coordinates. Although $Z$ and $Y_{c}$ are generally non-orthogonal, these coordinates are often used to parametrize the composition space for reaction zone analyses and we also adopt this representation here. Figure 2 schematically shows intersections of two exemplary mixture fraction gradient trajectories $(\mathrm{GT})$ and two $Z$-isolines in a two-dimensional 
plane. It is assumed that the shaded region is located in the vicinity of a reaction zone, i.e. an area with significant chemical activity and heat release. Traversing points along a gradient trajectory in the physical space (i.e. constructing a trajectory following $\nabla Z$ ), both mixture fraction and progress variable change, which results in a corresponding motion in composition space (red arrow). In case there are no progress variable gradients along $Z$-isolines (i.e. $\nabla_{\perp} Y_{c}=0$ ) this motion is analogous to the traversal of a non-premixed flame. On the contrary, traversing points along $Z$-isolines (i.e. along $\nabla_{\perp} Y_{c}$ ) results in a motion in composition space along $Y_{c}$ only (blue arrow), while $Z$ remains constant. If mixture gradients are sufficiently small this motion corresponds to the traversal of a one-dimensional premixed flame (neglecting differential diffusion).

\subsection{Composition space equations for species and temperature}

Introducing Eq. 16] into Eq. 6 and multiplying by the density yields

$$
\begin{aligned}
\rho \frac{\partial Y_{i}}{\partial \tau} & =\rho D \nabla Z \cdot \nabla\left(\frac{\partial Y_{i}}{\partial Z}\right)+\rho D \nabla_{\perp} Y_{c} \cdot \nabla\left(\left.\frac{\partial Y_{i}}{\partial Y_{c}}\right|_{Z}\right)+\left.\nabla \cdot\left(\rho D \nabla_{\perp} Y_{c}\right) \frac{\partial Y_{i}}{\partial Y_{c}}\right|_{Z}+\dot{\omega}_{i} \\
& =\rho D|\nabla Z|^{2} \frac{\partial^{2} Y_{i}}{\partial Z^{2}}+\left.\rho D\left|\nabla_{\perp} Y_{c}\right|^{2} \frac{\partial^{2} Y_{i}}{\partial Y_{c}^{2}}\right|_{Z}+\left.\nabla \cdot\left(\rho D \nabla_{\perp} Y_{c}\right) \frac{\partial Y_{i}}{\partial Y_{c}}\right|_{Z}+\dot{\omega}_{i}
\end{aligned}
$$

In the expression above, Eq. 15) was used to reformulate the first and second term on the right hand side according to

$$
\begin{aligned}
\rho D \nabla Z \cdot \nabla\left(\frac{\partial Y_{i}}{\partial Z}\right) & =\rho D|\nabla Z|\left(\mathbf{n}_{1} \cdot \mathbf{n}_{1}\right)|\nabla Z| \frac{\partial}{\partial Z}\left(\frac{\partial Y_{i}}{\partial Z}\right) \\
& +\left.\rho D|\nabla Z|\left(\mathbf{n}_{1} \cdot \mathbf{n}_{2}\right)\left|\nabla_{\perp} Y_{c}\right| \frac{\partial}{\partial Y_{c}}\right|_{Z}\left(\frac{\partial Y_{i}}{\partial Z}\right) \\
& =\rho D|\nabla Z|^{2} \frac{\partial^{2} Y_{i}}{\partial Z^{2}},
\end{aligned}
$$

where $\mathbf{n}_{1} \cdot \mathbf{n}_{1}=1$ and $\mathbf{n}_{1} \cdot \mathbf{n}_{2}=0$ (orthogonality) was used.

With the intention to replace the third term on the right hand side of Eq. (18), a summation analogous to Eq. (12) is applied to Eq. (18)

$$
\rho \frac{\partial Y_{c}}{\partial \tau}=\rho D|\nabla Z|^{2} \frac{\partial^{2} Y_{c}}{\partial Z^{2}}+\rho D\left|\nabla_{\perp} Y_{c}\right|^{2} \underbrace{\left.\frac{\partial^{2} Y_{c}}{\partial Y_{c}^{2}}\right|_{Z}}_{=0}+\nabla \cdot\left(\rho D \nabla_{\perp} Y_{c}\right) \underbrace{\left.\frac{\partial Y_{c}}{\partial Y_{c}}\right|_{Z}}_{=1}+\dot{\omega}_{c}
$$

\footnotetext{
${ }^{1}$ As stated below Eq. 17 , partial derivatives with respect to $e$ are assumed small and omitted.
} 
Thus, Eq. (20) simplifies to

$$
\rho \frac{\partial Y_{c}}{\partial \tau}=\nabla \cdot\left(\rho D \nabla_{\perp} Y_{c}\right)+\dot{\omega}_{c_{\perp}}
$$

with the source

$$
\dot{\omega}_{c_{\perp}}=\rho D|\nabla Z|^{2} \frac{\partial^{2} Y_{c}}{\partial Z^{2}}+\dot{\omega}_{c}
$$

Equation (21) balances the progress variable $Y_{c}$ on $Z$-isosurfaces. Therefore, transport along the direction of $Z$-gradients (i.e. between different $Z$-isosurfaces) is attributed to the source term in Eq. 222). This term can also be interpreted as a correction term which ensures orthogonality between $\nabla Z$ and the newly introduced $\nabla_{\perp} Y_{c}$. From another perspective, the above expression for the source term is equivalent to the equation for an unsteady, non-premixed laminar flamelet [13] and in this context $\dot{\omega}_{c_{\perp}}$ corresponds to the transient term. This already indicates a suitable mapping between time and reaction progress variable, which has already been utilized for unsteady flamelet modeling [35] and modeling of homogeneous reacting mixtures [21, 29] in previous works. This aspect is revisited and discussed further below.

In a CFD simulation, it would be impractical to solve Eq. (21) since this would require $Z$-isosurfaces to be tracked in time and space. However, the equation is used to replace the third term on the right hand side of Eq. (18) which yields

$$
\rho \frac{\partial Y_{i}}{\partial \tau}=\rho D|\nabla Z|^{2} \frac{\partial^{2} Y_{i}}{\partial Z^{2}}+\left.\rho D\left|\nabla_{\perp} Y_{c}\right|^{2} \frac{\partial^{2} Y_{i}}{\partial Y_{c}^{2}}\right|_{Z}+\left.\rho \frac{\partial Y_{c}}{\partial \tau} \frac{\partial Y_{i}}{\partial Y_{c}}\right|_{Z}-\left.\dot{\omega}_{c_{\perp}} \frac{\partial Y_{i}}{\partial Y_{c}}\right|_{Z}+\dot{\omega}_{i}
$$

This equation is simplified assuming that the thermochemical state can be approximated by a reduced order manifold, which is parametrized by mixture fraction and progress variable. Then, a first order taylor series expansion of the first term in Eq. (23) can be written as

$$
\frac{\partial Y_{i}}{\partial \tau} \approx \underbrace{\frac{\partial Z}{\partial \tau}}_{=0} \frac{\partial Y_{i}}{\partial Z}+\left.\frac{\partial Y_{c}}{\partial \tau} \frac{\partial Y_{i}}{\partial Y_{c}}\right|_{Z}=\left.\frac{\partial Y_{c}}{\partial \tau} \frac{\partial Y_{i}}{\partial Y_{c}}\right|_{Z},
$$

which states that the transient evolution of the thermochemical state at the particle position (i.e. at constant mixture fraction) is related to the transient evolution of the progress variable at this point. Introducing the expression into Eq. (23) the transient terms compensate each other and vanish. It has been shown in previous works [28, 29] that composition space models can accurately recover characteristics of transient combustion processes (e.g. auto-ignition, spherical expanding flames) based on this approximation. This also applies to complex transient processes such as two-stage ignition [29]. Equation (24) is based on the fact that chemical reaction mechanisms propagate the change in one species through a network 
of chemical reactions to many others. Thereby, only few species, which are directly or indirectly affected by the most relevant reaction pathways, are required in the definition of $Y_{c}$ (c.f. Eq. (12)). Nevertheless, it is noted that Eq. (24) can lead to modeling errors for very large transients experienced by certain intermediate species, such as radicals.

Introducing Eq. (24) into Eq. (23) and performing an analogous transformation for the temperature, which is subject to the transport equation (unity Lewis numbers)

$$
\rho \frac{\partial T}{\partial t}+\rho \mathbf{u} \cdot \nabla T=\frac{1}{c_{p}} \nabla \cdot(\lambda \nabla T)+\sum_{k}^{n_{s}} \frac{c_{p, k}}{c_{p}} \rho D \nabla Y_{k} \cdot \nabla T+\dot{\omega}_{T},
$$

yields the two-dimensional composition space equations

$$
\begin{aligned}
&\left.\dot{\omega}_{c_{\perp}} \frac{\partial T}{\partial Y_{c}}\right|_{Z}=\rho D|\nabla Z|^{2} \frac{\partial^{2} T}{\partial Z^{2}}+\left.\rho D\left|\nabla_{\perp} Y_{c}\right|^{2} \frac{\partial^{2} T}{\partial Y_{c}^{2}}\right|_{Z} \\
&+\frac{\rho D}{c_{p}}\left(|\nabla Z|^{2} \frac{\partial c_{p}}{\partial Z} \frac{\partial T}{\partial Z}+\left.\left.\left|\nabla_{\perp} Y_{c}\right|^{2} \frac{\partial c_{p}}{\partial Y_{c}}\right|_{Z} \frac{\partial T}{\partial Y_{c}}\right|_{Z}\right) \\
&+\rho D \sum_{k}^{n_{s}} \frac{c_{p, k}}{c_{p}}\left(|\nabla Z|^{2} \frac{\partial Y_{k}}{\partial Z} \frac{\partial T}{\partial Z}+\left.\left.\left|\nabla_{\perp} Y_{c}\right|^{2} \frac{\partial Y_{k}}{\partial Y_{c}}\right|_{Z} \frac{\partial T}{\partial Y_{c}}\right|_{Z}\right)+\dot{\omega}_{T} \\
& \underbrace{\left.\dot{\omega}_{c_{\perp}} \frac{\partial Y_{i}}{\partial Y_{c}}\right|_{Z}}_{Y_{c} \text {-drift }}=\underbrace{\rho D|\nabla Z|^{2} \frac{\partial^{2} Y_{i}}{\partial Z^{2}}}_{Z \text {-diffusion }}+\underbrace{\left.\rho D\left|\nabla_{\perp} Y_{c}\right|^{2} \frac{\partial^{2} Y_{i}}{\partial Y_{c}^{2}}\right|_{Z}}_{Y_{c}-\text { diffusion }}+\underbrace{\dot{\omega}_{i}}_{\text {source }} .
\end{aligned}
$$

Considering the species equation above, it consists of a drift term, two diffusive transport terms along the directions of $Z$ and (orthogonal) $Y_{c}$, respectively, and a chemical source term. The drift term, formally a convective term in composition space, incorporates the evolution of the reaction progress variable itself. An equivalent term does not exist for the mixture fraction since it is a passive scalar. Opposed to similar previous works [11, 22, 36], Eqs. (26) and (27) do not contain so-called cross-terms due to orthogonality of the employed coordinate system. Comparing the equation set to the one presented by Nguyen et al. [22] (c.f. Eqs. (21) and (22) of their work), who conditioned temperature and species on $\left(Z, Y_{c}\right)$ without explicitly incorporating orthogonality, the two main differences besides the removal of cross-terms are: (1) the correction for the progress variable source term $\dot{\omega}_{c_{\perp}}$ (c.f. Eq. (22)), and (2) the modified gradient $\left|\nabla_{\perp} Y_{c}\right|$ scaling diffusion along the $Y_{c^{-}}$-dimension (keeping $Z$ fixed). The absence of cross-terms is examined further in Sec. 2.5. Before, it is demonstrated that the equations recover the asymptotic limits of combustion.

\subsection{Recovery of limiting cases of combustion}

The composition space equations describing (i) a homogeneous reacting mixture, (ii) a premixed flamelet, and (iii) a non-premixed flamelet can be readily obtained as a subset of 
Eqs. 26) and (27).

Homogeneous reacting mixture. In the absence of spatial gradients $\left(|\nabla Z|=\left|\nabla_{\perp} Y_{c}\right|=0\right)$, the mixture fraction is uniform $\left(Z=\right.$ const.). In this case, the balance equation for $Y_{c}$ on $Z$-isosurfaces, Eq. 21, becomes equivalent to the $Y_{c}$-equation, Eq. (8), and the homogeneous reactive mixture only evolves in time. Equations (26) and (27) simplify to

$$
\dot{\omega}_{c} \frac{\partial T}{\partial Y_{c}}=\dot{\omega}_{T} \quad \text { and } \quad \dot{\omega}_{c} \frac{\partial Y_{i}}{\partial Y_{c}}=\dot{\omega}_{i}
$$

It has been shown [21, 29] that these equations accurately describe the evolution of a constant pressure homogeneous reacting mixture (ignition problem) in composition space. Note that a time-like coordinate in composition space is not required since the unsteadiness of thermochemical variables in the physical space is inherently captured through the transient evolution of the conditioning variables (here only of $Y_{c}$ ), c.f. Eq. (24).

Premixed flamelet. In the limit of a premixed flame (assuming Le $=1$ ), the mixture fraction gradient is zero, $\nabla Z=0$. From Eq. (13) it is obvious that $\nabla_{\perp} Y_{c}=\nabla Y_{c}$. Furthermore, the correction term vanishes in Eq. (22) and $\dot{\omega}_{c_{\perp}}=\dot{\omega}_{c}$.

In composition space, the Eqs. (26) and (27) consistently simplify to the 1D premixed flamelet equations [28, 34, 37]:

$$
\begin{aligned}
\dot{\omega}_{c} \frac{\partial T}{\partial Y_{c}} & =\rho D\left|\nabla Y_{c}\right|^{2} \frac{\partial^{2} T}{\partial Y_{c}^{2}}+\frac{\rho D}{c_{p}}\left|\nabla Y_{c}\right|^{2} \frac{\partial c_{p}}{\partial Y_{c}} \frac{\partial T}{\partial Y_{c}} \\
& +\rho D \sum_{k}^{n_{s}} \frac{c_{p, k}}{c_{p}}\left|\nabla Y_{c}\right|^{2} \frac{\partial Y_{k}}{\partial Y_{c}} \frac{\partial T}{\partial Y_{c}}+\dot{\omega}_{T}, \\
\dot{\omega}_{c} \frac{\partial Y_{i}}{\partial Y_{c}} & =\rho D\left|\nabla Y_{c}\right|^{2} \frac{\partial^{2} Y_{i}}{\partial Y_{c}^{2}}+\dot{\omega}_{i} .
\end{aligned}
$$

Non-premixed flamelet. For a purely non-premixed flame, $Y_{c}$ can be expressed as a function of $Z$ which implies that gradients $\nabla Z$ and $\nabla Y_{c}$ are aligned. It follows for Eq. (13)

$$
\nabla_{\perp} Y_{c}=\nabla Y_{c}-\frac{\partial Y_{c}}{\partial Z} \nabla Z=0
$$

Equation 16 then reduces to the conventional flamelet transformation rule $\nabla T=\nabla Z \frac{\partial T}{\partial Z}$ and the balance equation for $Y_{c}$ on $Z$-isosurfaces, Eq. 21, simplifies to

$$
\rho \frac{\partial Y_{c}}{\partial \tau}=\dot{\omega}_{c_{\perp}}=\rho D|\nabla Z|^{2} \frac{\partial^{2} Y_{c}}{\partial Z^{2}}+\dot{\omega}_{c}
$$


Accordingly, one obtains from Eqs. 26 and 27)

$$
\begin{aligned}
\left.\dot{\omega}_{c_{\perp}} \frac{\partial T}{\partial Y_{c}}\right|_{Z} & =\rho D|\nabla Z|^{2} \frac{\partial^{2} T}{\partial Z^{2}}+\frac{\rho D}{c_{p}}|\nabla Z|^{2} \frac{\partial c_{p}}{\partial Z} \frac{\partial T}{\partial Z} \\
& +\rho D \sum_{k}^{n_{s}} \frac{c_{p, k}}{c_{p}}|\nabla Z|^{2} \frac{\partial Y_{k}}{\partial Z} \frac{\partial T}{\partial Z}+\dot{\omega}_{T}, \\
\left.\dot{\omega}_{c_{\perp}} \frac{\partial Y_{i}}{\partial Y_{c}}\right|_{Z} & =\rho D|\nabla Z|^{2} \frac{\partial^{2} Y_{i}}{\partial Z^{2}}+\dot{\omega}_{i} .
\end{aligned}
$$

It is readily shown that the above equation set is equivalent to the unsteady 1D flamelet equations for non-premixed combustion [13] by replacing the drift term on the left hand side according to

$$
\left.\left.\dot{\omega}_{c_{\perp}} \frac{\partial Y_{k}}{\partial Y_{c}}\right|_{Z} \stackrel{32}{=} \rho \frac{\partial Y_{c}}{\partial \tau} \frac{\partial Y_{k}}{\partial Y_{c}}\right|_{Z} \stackrel{24}{=} \rho \frac{\partial Y_{k}}{\partial \tau} .
$$

This illustrates again the relationship between time and the reaction progress variable. Note that if only the mixture fraction $Z$ was chosen as a conditioning variable (without a suitable coordinate capturing reaction progress), the transient term remains in the equations during the derivation presented in Sec. 2.3 .

\subsection{Discussion of conceivable closures, cross-terms and differential diffusion}

The composition space theory presented so far can be used for analyses of fully resolved numerical datasets (c.f. Sec. 3). Further, an algorithm for the numerical solution of Eqs. (26) and (27) would be very valuable for modeling multi-regime combustion. A reduced order manifold could thereby be constructed directly in composition space and tabulated as a function of a reduced set of scalars (mixture fraction, progress variable and others). While such an algorithm is subject to future research, three key issues for this effort are briefly discussed here: (i) closures for the gradients $|\nabla Z|$ and $\left|\nabla_{\perp} Y_{c}\right|$, (ii) cross-terms, and (iii) an extension to capture differential diffusion (non-unity Lewis numbers).

As stated above, a numerical solution algorithm for Eqs. (26) and (27) requires closures for $|\nabla Z|$ and $\left|\nabla_{\perp} Y_{c}\right|$. From the analytical solution of mixing layers [38] promising closures exist for $|\nabla Z|$ (or the scalar dissipation rate $\chi_{Z}=2 D|\nabla Z|^{2}$ ). On the contrary, $\left|\nabla_{\perp} Y_{c}\right|$ depends on the progress variable gradient $\left|\nabla Y_{c}\right|$ which substantially changes with the fuel-oxidizer combination or the specific progress variable definition (c.f. Eq. (12) [28] and no generic analytical closure exists. In our previous works [28, 29], a separate equation for the progress variable gradient has been utilized and it is likely that this approach can be extended to complement the composition space equations presented here. This aspect should be explored in future work.

Further, comparing Eqs. (26) and (27) to the equations derived by Nguyen et al. [22], it is apparent that thermochemical quantities are evolving in four dimensions here, 
i.e. $Y_{i}\left(Z,\left.Y_{c}\right|_{Z},|\nabla Z|,\left|\nabla_{\perp} Y_{c}\right|\right)$, opposed to five dimensions in [22], i.e. $Y_{i}\left(Z, Y_{c}, \chi_{Z}, \chi_{Y_{c}}, \chi_{Z, Y_{c}}\right)$. While $|\nabla Z|$ is readily related to the scalar dissipation rate $\chi_{Z}=2 D|\nabla Z|^{2},\left|\nabla_{\perp} Y_{c}\right|$ is linked to both $\chi_{Y_{c}}=2 D\left|\nabla Y_{c}\right|^{2}$ and the cross-scalar dissipation rate $\chi_{Z, Y_{c}}=2 D \nabla Z \cdot \nabla Y_{c}$. Without orthogonal coordinates, cross-terms appear in composition space equations [11, 22, 36] with $\chi_{Z, Y_{c}}$ as a scaling factor. Cross-terms require a closure and are difficult to handle from a modeling perspective. So far no general closure strategy has been presented and sometimes these terms are neglected accepting a certain modeling error. The cross-scalar dissipation rate $\chi_{Z, Y_{c}}$ is defined based on the scalar product

$$
\nabla Z \cdot \nabla Y_{c}=\frac{\chi_{Z, Y_{c}}}{2 D}=|\nabla Z|^{2} \frac{\partial Y_{c}}{\partial Z}=|\nabla Z|\left|\nabla Y_{c}\right| \cos \varphi
$$

where $\varphi$ is the enclosed angle between the gradients $\nabla Z$ and $\nabla Y_{c}$. Furthermore, $\left|\nabla_{\perp} Y_{c}\right|$ is related to $\left|\nabla Y_{c}\right|$ through $\varphi$

$$
\begin{aligned}
\left|\nabla_{\perp} Y_{c}\right|^{2} & =\nabla_{\perp} Y_{c} \cdot \nabla_{\perp} Y_{c}=\nabla_{\perp} Y_{c} \cdot\left(\nabla Y_{c}-\nabla Z \frac{\partial Y_{c}}{\partial Z}\right) \\
& =\nabla_{\perp} Y_{c} \cdot \nabla Y_{c}=\left|\nabla Y_{c}\right|^{2}-\nabla Z \cdot \nabla Y_{c} \frac{\partial Y_{c}}{\partial Z} \\
& =\left|\nabla Y_{c}\right|^{2}\left(1-\cos ^{2} \varphi\right),
\end{aligned}
$$

which is linked to the definition of the second coordinate $\mathbf{n}_{2}$ of the orthogonal coordinate system introduced with Eq. (10). Hence, the absence of cross-terms in Eqs. (26) and (27) is achieved by incorporating the interdependence between progress variable and mixture fraction into the construction of the orthogonal coordinates.

As previously stated, $Z$ and $Y_{c}$ are generally non-orthogonal coordinates. This affects the numerical solution of Eqs. (26) and (27): if the composition space equations (26) and (27) are discretized on a $\left(Z, Y_{c}\right)$-grid, which is for that purpose made orthogonal, partial derivatives with respect to $Z$ have to be split into a component keeping $Y_{c}$ constant and cross-terms are re-introduced as correction terms to account for the non-orthogonality of $\left(Z, Y_{c}\right)$. This issue remains beyond the scope of this paper, but it should be noted that the numerical solution of the composition space equations is therefore not trivial.

Nevertheless, the combination of $Z$ and $Y_{c}$ as conditioning variables for thermochemical scalars is a natural choice for combustion processes and these quantities should be kept to parametrize numerical solutions of Eqs. (26) and (27). In the context of tabulated chemistry approaches, already existing turbulence-chemistry interaction models, as required for LargeEddy Simulations, can be used with minor modifications. Even if the composition space equations are eventually solved in a different reference frame, mixture fraction and progress variable can be reconstructed for tabulation and look-up.

A worthwhile extension of the present composition space theory is the consistent represen- 
tation of differential diffusion (non-unity Lewis numbers). Previous works on one-dimensional composition space modeling for premixed [28, 29, 34] and non-premixed combustion [39, 40] have shown that this can be realized conditioning on either mixture fraction or progress variable. The extension of the two-dimensional composition space equations for differential diffusion requires a careful incorporation of detailed diffusion modeling leading to additional terms and transport effects, such as curvature-induced differential diffusion [41]. This extension for the Eqs. (26) and (27) is therefore non-trivial, but highly important for describing multi-regime combustion without restrictive assumptions regarding diffusive transport.

\section{Analysis of a laminar triple flame}

The composition space equations presented in the previous section are validated with the fully resolved, detailed chemistry solution of a laminar methane-air triple flame. Figure 3 shows a schematic of the triple flame setup. It consists of a lean and a rich premixed flame branch which enclose a non-premixed flame. The three different flame structures merge at the characteristic triple point, which plays an important role for the flame stabilization. The triple flame is established by defining an inflow of premixed fresh gases $\left(T_{0}=300 \mathrm{~K}, p=1 \mathrm{~atm}\right)$ with a mixture stratification in the cross-flow direction. The stoichiometric mixture fraction is $Z_{\mathrm{st}}=0.055$ (methane-air), and the boundary conditions are chosen as $Z_{\text {min }}=0$ (pure air) and $Z_{\max }=0.42$. The boundary conditions in the cross-flow direction are implemented as slip walls. The imposed mixture fraction gradient at the inlet is $50 \mathrm{~m}^{-1}$ which results in an edge-flame aspect ratio of $f=0.024$. The latter expresses a ratio between a diffusion length measured along the stoichiometric surface (premixed kernel) and a diffusion length across the stoichiometric surface (diffusion flame) [42]. With $f=0.024$, the laminar triple flame corresponds to a weakly curved partially premixed flame according the classification introduced in [42]. The GRI-MECH 3.0 reaction mechanism [43] is used for describing chemical kinetics and unity Lewis numbers are assumed for all species. Further information on the numerical setup of the triple flame can be found in [12]. From the fully resolved scalar

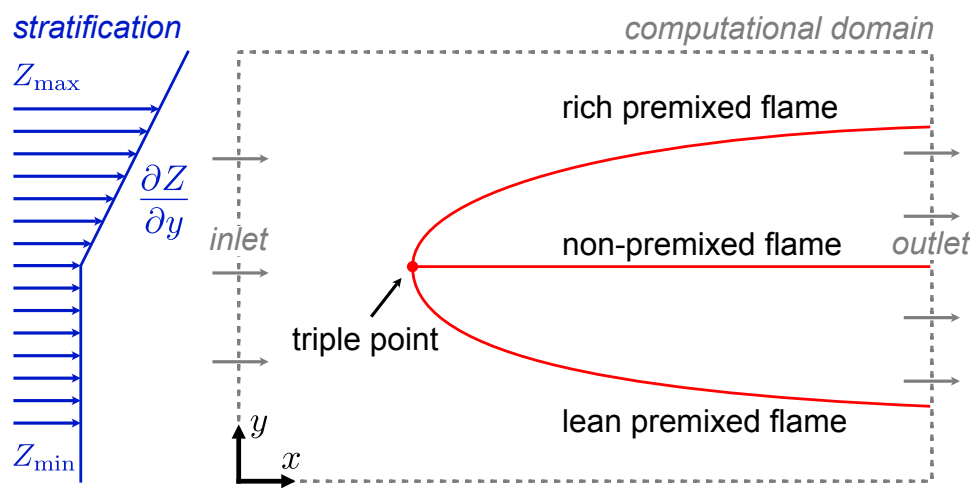

Figure 3: Schematic of the laminar triple flame. The flame is established by a mixture stratification (imposed mixture fraction gradient) of the fresh gases at the inlet boundary. 
fields of this flame all terms which appear in the 2D composition space equations, Eqs. (26) and (27), are reconstructed and evaluated.

\subsection{Triple flame characteristics}

Simulation results obtained for the scalar fields of temperature, heat release rate (HRR) and four species mass fractions of the laminar triple flame are displayed in Fig. 4. The largest heat release rate is found in the vicinity of the triple point, where the conversion of reactants (c.f. $\mathrm{O}_{2}$ ) to hot products (c.f. $\mathrm{CO}_{2}$ ) and the maximum heat release occur in a thin reactive layer. Further, radical species, such as $\mathrm{OH}$, are found to reach a maximum there. The thickness of the reactive layer, which can be assessed from the transition region of reactants to products, increases downstream of the triple point marking the two premixed flame branches. The upper rich premixed flame appears stronger than the lower lean premixed flame since its contribution to the overall heat release is larger. The heat release by the non-premixed flame remains at least one order of magnitude smaller than that of the premixed flames which is why it remains hardly visible in the HRR plot. As expected, considerably more CO is formed in the rich premixed flame compared to the lean side of the triple flame. The overall maximum of temperature and reaction products ( such as $\mathrm{CO}_{2}$ ) is eventually reached at the position of the non-premixed flame, where conditions approach the chemical equilibrium.
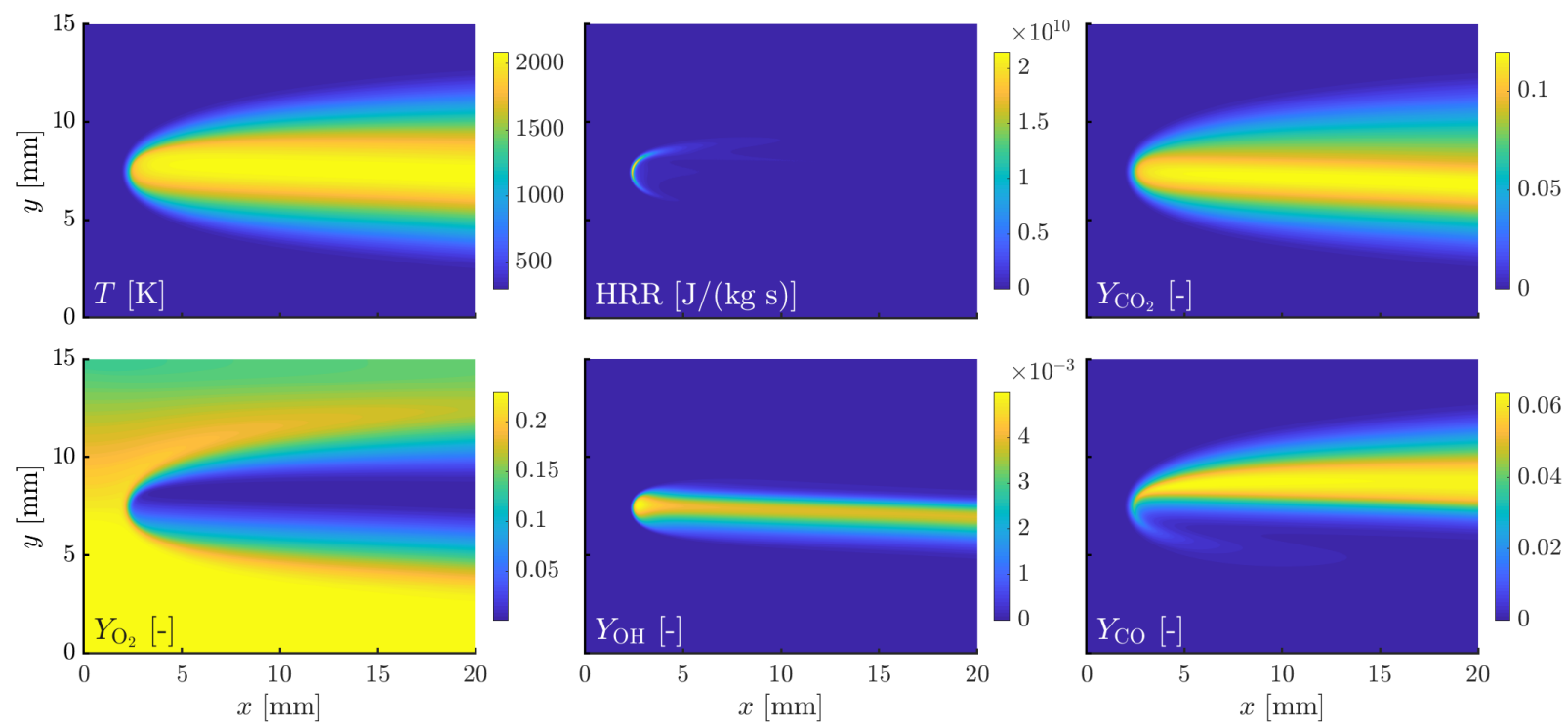

Figure 4: Scalar fields of temperature, heat release rate (HRR) and mass fractions of the species $\mathrm{CO}_{2}, \mathrm{O}_{2}$, $\mathrm{OH}$ and $\mathrm{CO}$ for the triple flame.

Figure 5 shows the scalar fields of the conditioning variables $Z$ and $Y_{c}$. The scalar mixture fraction field illustrates the stratification along the $y$-direction which remains effective over the whole domain. The reaction progress has a very similar structure as a chemical product species, which is readily anticipated from its definition $Y_{c}=Y_{\mathrm{H}_{2} \mathrm{O}}+Y_{\mathrm{CO}_{2}}$. In the top right plot, the source term $\dot{\omega}_{c_{\perp}}$ is shown. Furthermore, the figure displays the gradients $|\nabla Z|$, 

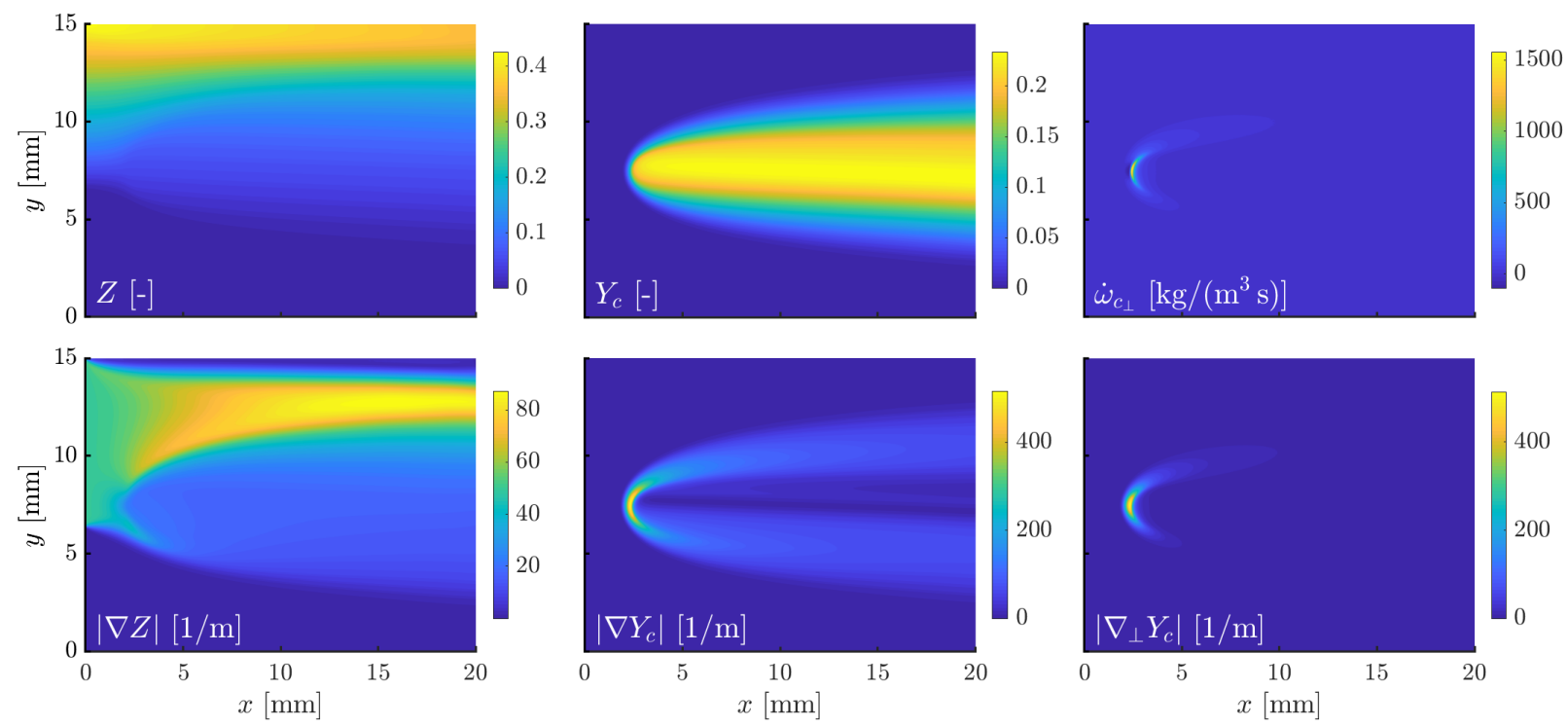

Figure 5: Scalar fields of the conditioning variables $Z$ and $Y_{c}$, the source term $\dot{\omega}_{c_{\perp}}$ (top row) together with the gradients $|\nabla Z|,\left|\nabla Y_{c}\right|$ and $\left|\nabla_{\perp} Y_{c}\right|$ (bottom row). The progress variable is defined as $Y_{c}=Y_{\mathrm{H}_{2} \mathrm{O}}+Y_{\mathrm{CO}_{2}}$.

$\left|\nabla Y_{c}\right|$ and $\left|\nabla_{\perp} Y_{c}\right|$ in the bottom row. The mixture fraction gradient $|\nabla Z|$ indicates intense mixing for the rich premixed flame branch and in the vicinity of the triple point, where also the highest chemical activity is perceived (c.f. HRR in Fig. 4). From the gradient $\left|\nabla Y_{c}\right|$, which assumes large magnitudes at premixed flame fronts, both premixed flame branches can be identified. Additionally, zero values of $\left|\nabla Y_{c}\right|$, which appear mid-domain as a dark blue stripe, indicate the non-premixed flame. These flame fronts are less pronounced in the plot of $\left|\nabla_{\perp} Y_{c}\right|$, which represents the gradient component perpendicular to the mixture fraction gradient. It shows values of the same magnitude as $\left|\nabla Y_{c}\right|$ in the vicinity of the triple point and along a portion of the premixed flame branches but it assumes small values everywhere else. This is also reflected for the source term $\dot{\omega}_{c_{\perp}}$ which exhibits large values close to the triple point.

Similar characteristics are found from Fig. 6, which shows the angle between $\nabla Z$ and $\nabla Y_{c}$ (left) and gradient trajectories of $\nabla Z, \nabla Y_{c}$, and $\nabla_{\perp} Y_{c}$, respectively (middle and right). Note that the definition of the angle is slightly modified here

$$
\varphi^{*}=\arccos \left(\left|\frac{\nabla Z \cdot \nabla Y_{c}}{|\nabla Z|\left|\nabla Y_{c}\right|}\right|\right)
$$

such that $\varphi^{*}=0^{\circ}$ indicates a full alignment between $\nabla Z$ and $\nabla Y_{c}$ and $\varphi^{*}=90^{\circ}$ indicates orthogonality. From both, Figs. 6 (left) and 6 (middle), it is apparent that $\nabla Z$ and $\nabla Y_{c}$ are strongly misaligned $\left(\varphi^{*}=50 \ldots 90^{\circ}\right)$ upstream from and also directly at the position of the triple point. On the other hand, $\nabla Z$ and $\nabla Y_{c}$ become almost perfectly aligned $\left(\varphi^{*} \approx 0^{\circ}\right)$ for the downstream region $x>5 \mathrm{~mm}$. These characteristics suggest that $Z-Y_{c}$ cross-terms, corresponding to non-negligible values for $0^{\circ}<\varphi^{*}<90^{\circ}$ and significant gradients of $|\nabla Z|$ 

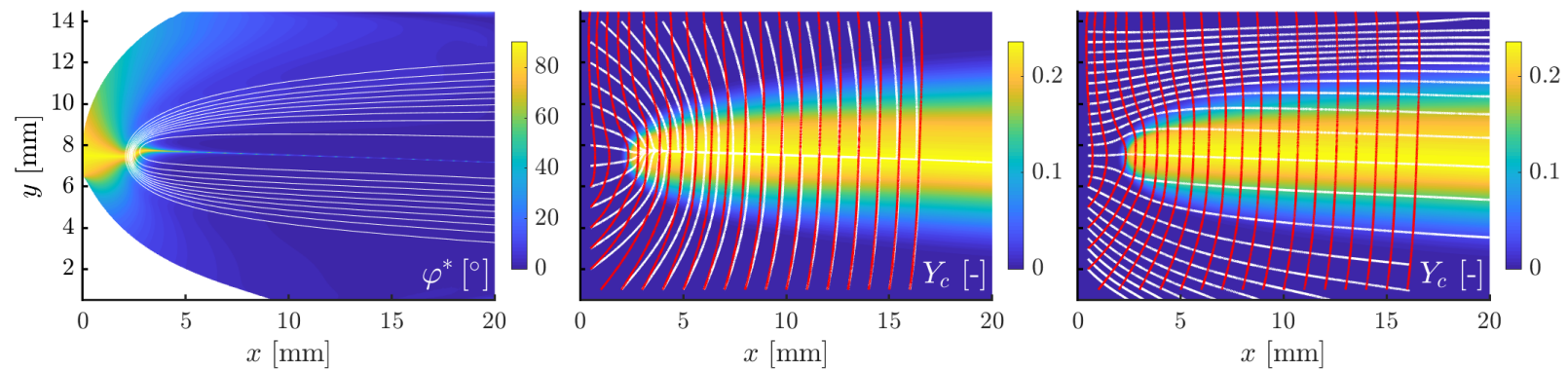

Figure 6: Left: angle between $\nabla Z$ and $\nabla Y_{c}$ (with overlay of white $Y_{c}$-isolines). Middle: $Y_{c}$-scalar field with an overlay of gradient trajectories for $\nabla Z$ (red) and $\nabla Y_{c}$ (white). Gradient trajectories are defined for a gradient field analogously to streamlines for a velocity vector field. Right: $Y_{c}$-scalar field with an overlay of gradient trajectories for $\nabla Z$ (red) and $\nabla_{\perp} Y_{c}$ (white).

and $\left|\nabla Y_{c}\right|$, play an important role for the stabilization mechanism and the most reactive region of the triple flame. According to the considerations in Sec. 2.4, these characteristics further imply multi-regime combustion due to deviations from the premixed (negligible $|\nabla Z|$ ) and the non-premixed $\left(\varphi^{*} \approx 0^{\circ}\right)$ burning mode. On the other hand, gradient alignment in the downstream region $(x>5 \mathrm{~mm})$ suggests that only one conditioning variable is necessary to describe local reaction zones as either the progress variable can be expressed as a function of the mixture fraction (non-premixed limit) or the mixture fraction gradient tends to zero (premixed limit). Additionally, the right plot displays gradient trajectories for $\nabla Z$ and $\nabla_{\perp} Y_{c}$ confirming that both gradients are perpendicular throughout the computational domain.

\subsection{Gradient-free regime analysis}

The gradient-free regime identification (GFRI) approach [3, 12] allows the identification of the local combustion regime based on experimental and numerical data. Starting from the fully resolved numerical solution of the triple flame, the mixture fraction $Z$, the heat release rate (HRR), and the chemical mode (CM) are calculated and used to detect and characterize reaction zones. It was shown that strong correlations between the magnitude of change in the CM zero-crossing, the mixture fraction at the CM zero-crossing, and the local HRR at the CM zero-crossing exist [3]. Hence, the combination of $Z, \mathrm{HRR}$, and CM allows the identification of dominantly premixed, dominantly non-premixed, and intermediate structures in which both premixed and non-premixed reaction zones contribute to the overall heat release rate. Further, the approach was extended and an automated classification of local reaction zone structures in complex multi-regime flames was derived [4]. Based on the ratio of local HRR peaks associated with premixed $\left(\mathrm{HRR}_{\mathrm{P}}\right)$ and non-premixed $\left(\mathrm{HRR}_{\mathrm{NP}}\right)$ regions located in close spatial proximity, a classification into premixed, dominantly premixed, multi-regime, dominantly non-premixed, and non-premixed flame zones is possible by using the flame structure index $\eta$ :

$$
\eta=\frac{\mathrm{HRR}_{\mathrm{P}}-\mathrm{HRR}_{\mathrm{NP}}}{\mathrm{HRR}_{\text {max }}},
$$


where $\mathrm{HRR}_{\text {max }}$ is the HRR maximum in close spatial proximity of the local reaction zones. The criteria for the classification of reaction zones are summarized in Table 1.

Table 1: Criteria for the classification of reaction zones.

\begin{tabular}{lcc}
\hline Premixed & P & $1 \geq \eta>0.99$ \\
\hline Dominantly premixed & DP & $0.99 \geq \eta>0.8$ \\
\hline Multi-regime & MR & $0.8 \geq \eta>-0.8$ \\
\hline Dominantly non-premixed & DNP & $-0.8 \geq \eta>-1$ \\
\hline Non-premixed & NP & $\eta=-1$ \\
& & (no CM zero-crossing) \\
\hline
\end{tabular}

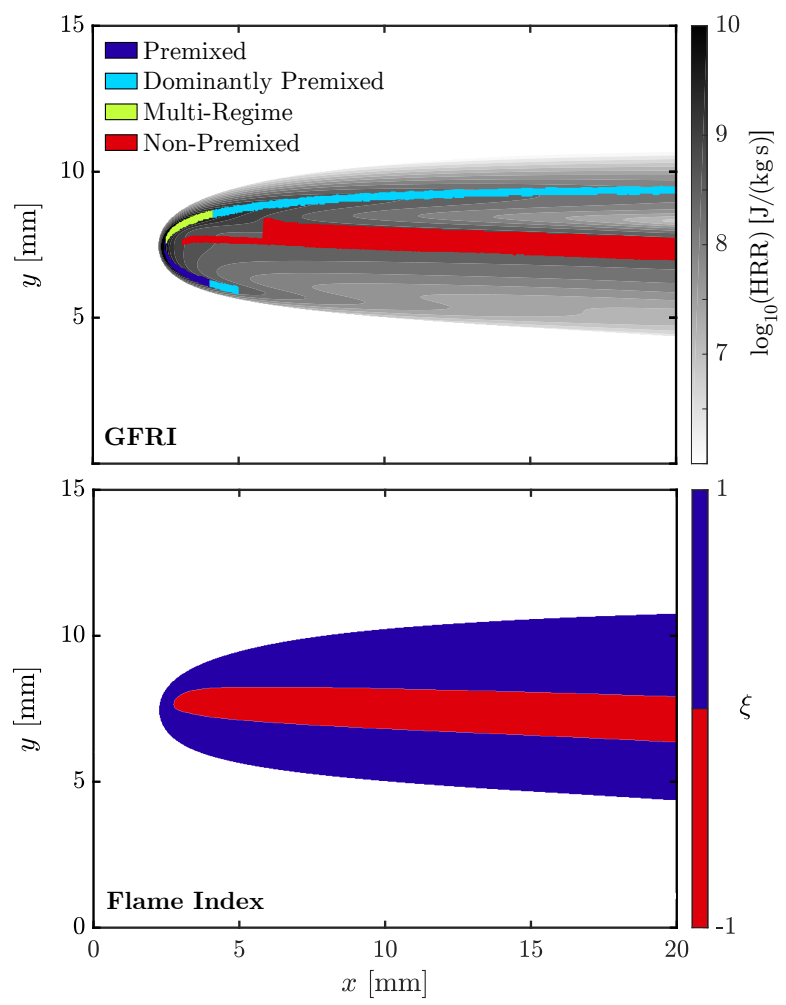

Figure 7: Top: Contour plot of the heat release rate (grey scales) and the distribution of local reaction zones according to the GFRI analysis. Bottom: normalized flame index $\xi$ evaluated from Eq. 400.

Figure 7 shows the distribution of the aforementioned combustion regimes for the laminar triple flame as a contour plot (top). From this figure the complex flame structure consisting of lean and rich premixed flame branches with an embedded non-premixed flame is visible. It is found that the intense HRR in the vicinity of the triple point is mainly driven by premixed, and multi-regime regions. Premixed and multi-regime structures exists until an axial position of $x=4 \mathrm{~mm}$ and are followed by dominantly premixed flame branches. These dominantly premixed flame zones are characterized by a maximum HRR which is shifted to the position of the CM zero-crossing, an indicator for slightly weakened premixed flames. 
Starting from $x=3 \mathrm{~mm}$, additionally non-premixed characteristics arise along the flame centerline until the end of the computational domain. It is emphasized that non-premixed structures also exist between the triple point and $x=3 \mathrm{~mm}$, however, the elevated local HRR values cannot be explicitly attributed to one specific flame zone due to the strong interaction between premixed, multi-regime and non-premixed zones. The lower premixed flame branch is characterized by the premixed and dominantly premixed flame region and the upper premixed flame branch by multi-regime and dominantly-premixed flame zones. Note, that multi-regime structures can only exist in rich to slightly rich regions, allowing premixed and non-premixed flame zones to exist in close proximity.

For reference, the normalized flame index is shown at the bottom of Fig. 7. It is evaluated from [10]

$$
\xi=\frac{\nabla Y_{\text {fuel }} \cdot \nabla Y_{\text {oxid }}}{\left|\nabla Y_{\text {fuel }} \cdot \nabla Y_{\text {oxid }}\right|}
$$

where $Y_{\text {fuel }}=Y_{\mathrm{CH}_{4}}$ and $Y_{\text {oxid }}=Y_{\mathrm{O}_{2}}$ denote the fuel and oxidizer mass fractions, respectively. From the flame index, premixed flames are identified by $\xi=1$ and non-premixed flames by $\xi=-1$. Overall, it agrees well with the results obtained from the GFRI approach, although it is evident that the latter yields more refined information on the individual flame zones.

The findings from the extended GFRI approach are in agreement with the analysis of the triple flame characteristics above and existing flame zone analyses from literature [11, 44]. It provides a more detailed understanding of the complex flame structures close to the triple point and serves as an orientation for the budget analysis of the $2 \mathrm{D}$ composition space equations in the following.

\subsection{Budget analysis of the 2D composition space equations}

A detailed analysis of the single terms of Eq. (27) for the species $\mathrm{CO}$ is performed. $\mathrm{CO}$ is chosen since it has been challenging to model this intermediate species in the context of tabulated chemistry approaches [4]. All terms which appear in the two-dimensional species equation are computed from the solution of the laminar triple flame using detailed chemistry. Such an analysis allows to quantify: (1) the local importance of the individual terms, and (2) confirms that all relevant transport effects are considered in the equations, i.e. that the transport terms balance the local chemical source term. It will be shown further below that this is not the case for conventional flamelet models relying on the assumption of a 1D flame structure. Figure 8 shows all terms of the species equation for $\mathrm{CO}$ along five different vertical slices in the vicinity of the triple point. For a perfectly balanced equation the sum of these terms is zero, which can be inspected in Fig. 8 by the term balance.

Slightly upstream of the triple point (1)), the chemical source term for CO is small and the dominant $Z$-diffusion (influence of stratification) is balanced by the $Y_{c}$-drift term. Closer to the triple point (2)), this characteristic prevails while the chemical source term and the $Y_{c}$-transport term both increase. Directly at the triple point (3)), the region with the highest chemical reactivity, the chemical source term becomes large and is compensated by all other terms, with the largest contribution from the $Y_{c}$-transport term. This observation agrees with 

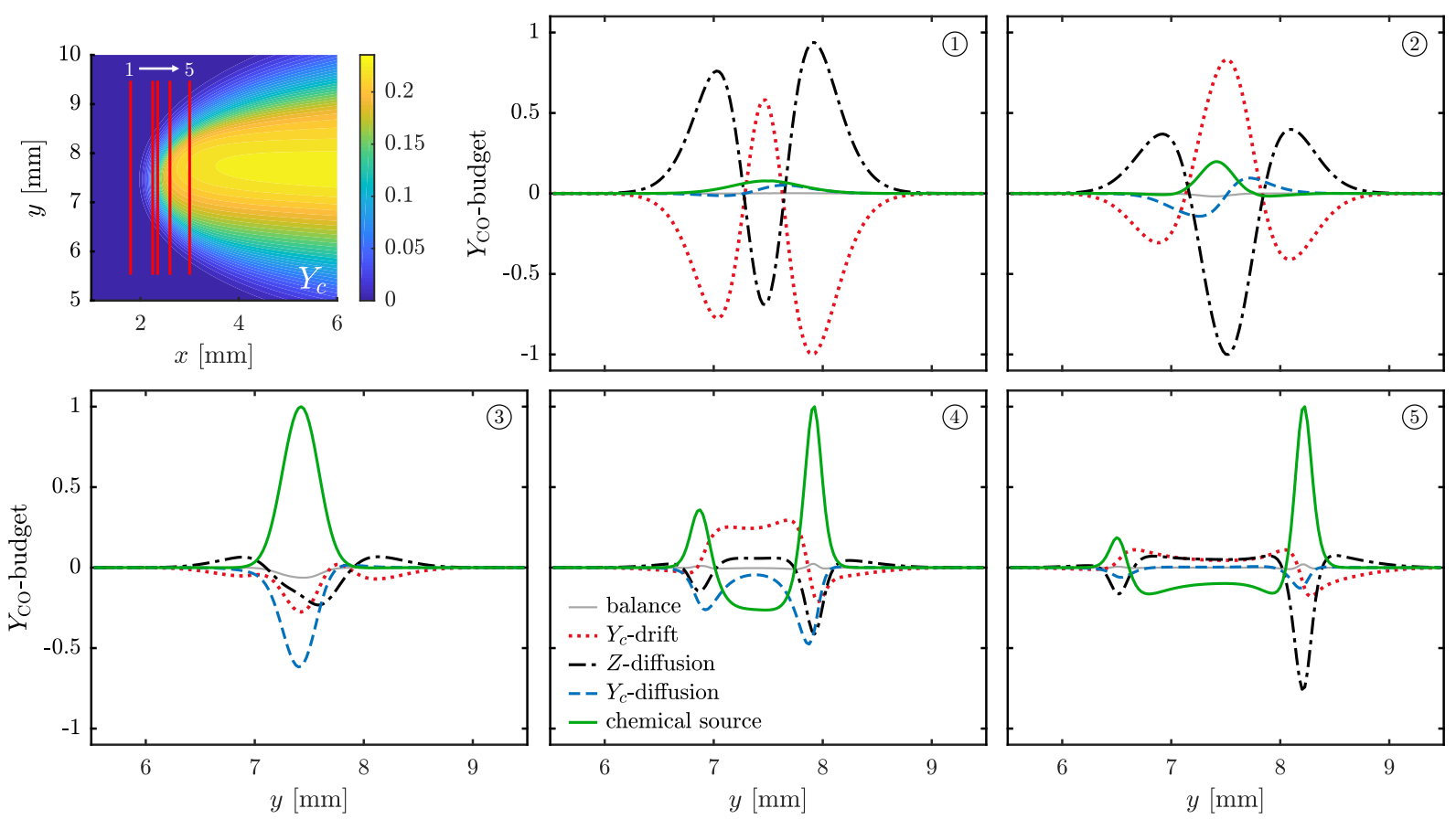

Figure 8: Normalized budgets of the species equation, Eq. (27), for $Y_{\mathrm{CO}}$ along five $x$-slices (vertical red lines in contour plot). All terms which appear in the $2 \mathrm{D}$ composition space equations were post-processed from the detailed chemistry solution of the triple flame.

the combustion regime identification which shows premixed and multi-regime characteristics close to the triple point. Further downstream (4) and (5)), two peaks of the chemical source terms form, which are related to the premixed flame branches. The chemical source term for the rich premixed flame is larger which reconfirms that this flame branch is stronger compared to the lean premixed flame. Notably, in the budgets of the composition space equations for slices (4) and (5) all terms are relevant and non-negligible. With this, Fig. 8 suggests that none of the terms in the equations is negligible for the triple flame and that the premixed flame branches downstream of the triple point are also significantly affected by stratification effects.

Figure 9 shows budgets of the 2D composition space equation for $\mathrm{CO}$ along horizontal slices of the triple flame. Five slices are extracted along the $y$-direction from upstream to downstream of the triple point. Slice 1 passes the lean premixed flame branch. It is found that at first the $Z$-diffusion and the $Y_{c}$-drift term balance each other upstream of the flame. Moving along the $x$-direction a positive peak in the chemical source term (CO-production region) is found and at that position additionally the $Y_{c}$-diffusion term gains relevance. The chemical source term then changes sign (CO-oxidation region) where the $Y_{c}$-diffusion becomes negligible small. Throughout this whole flame structure $Z$-diffusion and $Y_{c}$-drift are important. Considering the slices (2) and (3), which are located closer to the triple point, similar flame structures can be identified. Nevertheless, the structure becomes notably thinner, moves further upstream and the relative influence of $Z$-diffusion declines, while 

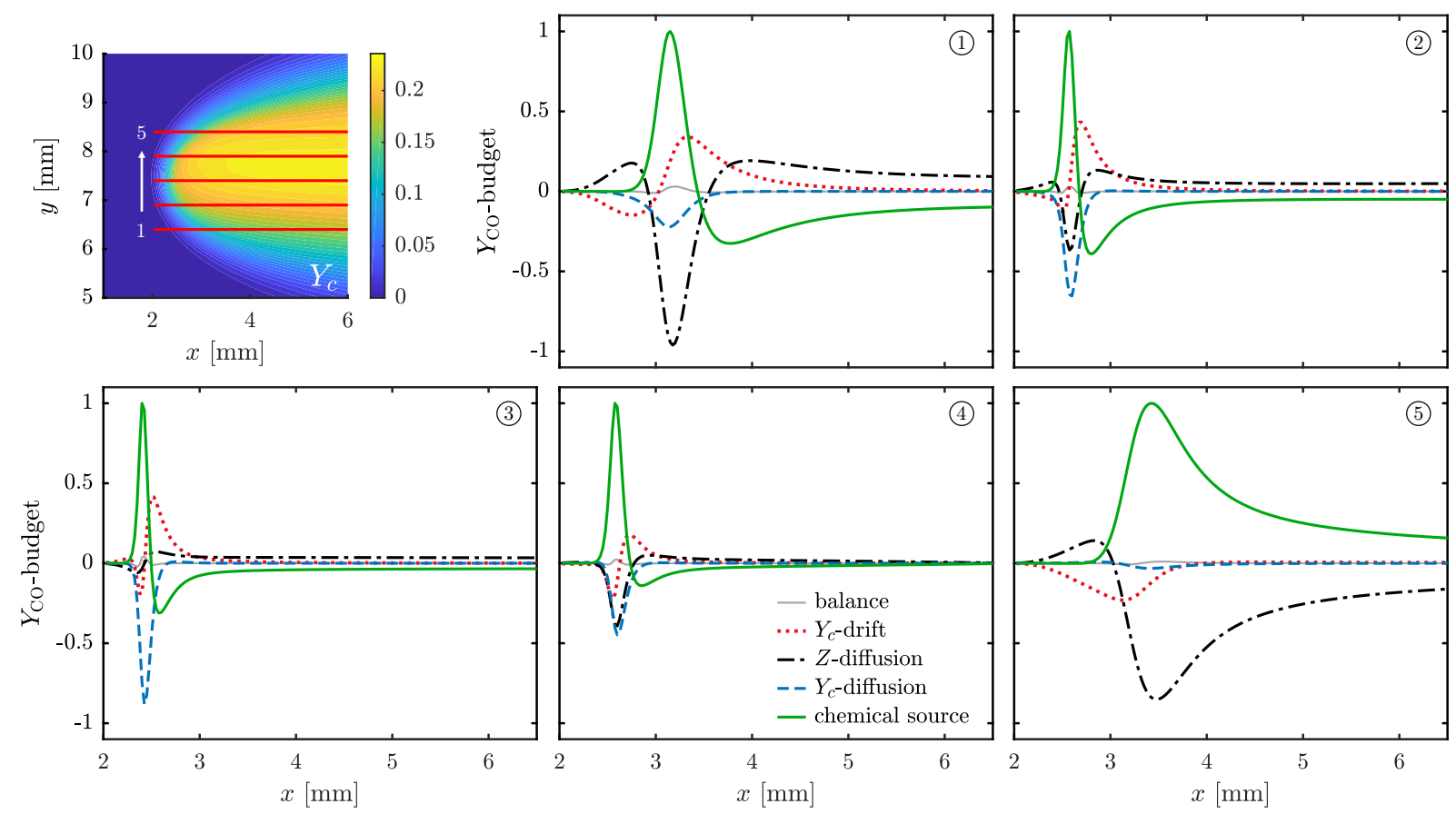

Figure 9: Normalized budgets of the species equation, Eq. 27), for $Y_{\mathrm{CO}}$ along several $y$-slices (horizontal red lines in contour plot). All terms which appear in the $2 \mathrm{D}$ composition space equations were post-processed from the detailed chemistry solution of the triple flame.

$Y_{c^{-}}$diffusion becomes a dominant term. Hence, according to the budgets the flame exhibits distinct premixed characteristics, which is in accordance with the previous findings. Above the triple point (4) and (5)), the $Z$-diffusion becomes more relevant again while $Y_{c}$-diffusion declines. Due to the rich conditions in the upper premixed flame branch (c.f. (5) the chemical source term remains positive and there is no further CO-oxidation to $\mathrm{CO}_{2}$.

Both, Figs. 8 and 9 show that the budgets of the 2D composition space equations closely reflect the regime characteristics also found from the GFRI analysis in Sec. 3.2. It becomes evident that different complex burning modes co-exist in the vicinity of the triple point which cannot solely be attributed to either premixed or non-premixed burning mode. Along these lines, Fig. 10 shows a comparison of the balance error of the 1D non-premixed flamelet equations (Eq. (34)), the 1D premixed flamelet equations (Eq. (30)) and the 2D composition space equations for multi-regime combustion (Eq. (27)). The equation balances are obtained from the summation of all terms which are post-processed from the detailed solution of the triple flame. Non-zero values indicate that the transport terms contained in the respective model do not fully balance the chemical source term. Note that the equation balances are normalized with the absolute value of the local chemical source term and they are only shown for points where chemical activity can be detected, i.e. where the source term exceeds $0.1 \%$ of its global maximum value. The left column in Fig. 10 shows that the non-premixed flamelet equations ( $Z$-flamelet) cannot capture the characteristics of the lean and rich premixed flame branches. On the other hand, the premixed flamelet equations ( $Y_{c}$-flamelet) in the middle 
column show substantial balance errors for $\mathrm{H}_{2} \mathrm{O}, \mathrm{CO}$ and $\mathrm{OH}$ downstream of the triple point. As indicated by the color scheme, these balance errors can become of the same order of magnitude as the chemical source term. While the conventional 1D flamelet models show large balance errors in certain regions, the $2 \mathrm{D}$ composition space equations are accurate everywhere throughout the triple flame. Hence, both asymptotic modes of burning as well as their complex interactions are recovered by the 2D composition space model.

\section{Summary and conclusions}

In this work, a new set of two-dimensional composition space equations for modeling multi-regime combustion is derived. Thermochemical variables are conditioned on the mixture fraction $Z$ and the reaction progress variable $Y_{c}$. While the orientation of the first coordinate is defined by the mixture fraction gradient, the second coordinate direction is based on the gradient component of the progress variable that is perpendicular to $\nabla Z$. This way, the interdependence between mixture fraction and progress variable is accounted for. The orthogonal set of coordinates is then used in the transformation of species and temperature equations to the two-dimensional composition space. Given certain simplifications, it is shown that the equations naturally reduce to conventional one-dimensional composition space equations for premixed and non-premixed combustion, and a homogeneous reacting mixture. Previous works have used non-orthogonal coordinates which lead to so-called cross-terms in the corresponding multi-dimensional composition space equations. These cross-terms require a closure, but to the authors' knowledge no general closure has been presented yet, if it exists at all. When using the orthogonal coordinates, cross-terms formally disappear.

In the second part of the paper, the two-dimensional composition space equations are validated with the detailed chemistry solution of a laminar methane-air triple flame. Postprocessing of this dataset allows the evaluation and examination of all terms appearing in the composition space equations. Together with a suitable combustion regime identification, it is shown that the novel equations accurately recover the balance of temperature and species everywhere in the triple flame. While conventional one-dimensional composition space equations fail particularly in the vicinity of the triple point, where multi-regime characteristics are detected, the novel two-dimensional equation set balances the chemical source term accurately. Moreover, the misalignment between mixture fraction and progress variable gradients suggest that $Z-Y_{c}$ cross-terms play a role in the stabilization mechanism and the flame structure around the triple point of the flame. This indicates that cross-terms are most likely non-negligible for modeling the multi-regime characteristics of the triple flame.

The theory presented in this work can be used for the analysis of fully-resolved numerical datasets of multi-regime combustion phenomena. However, for the method to reach its full potential, a numerical algorithm for solving the two-dimensional composition space equations is required. Therefore, two aspects should be investigated in future works: (1) closures for the gradients of the conditioning variables, which properly incorporate the orthogonality of the coordinates, and (2) identification of a robust numerical solution algorithm for the composition 

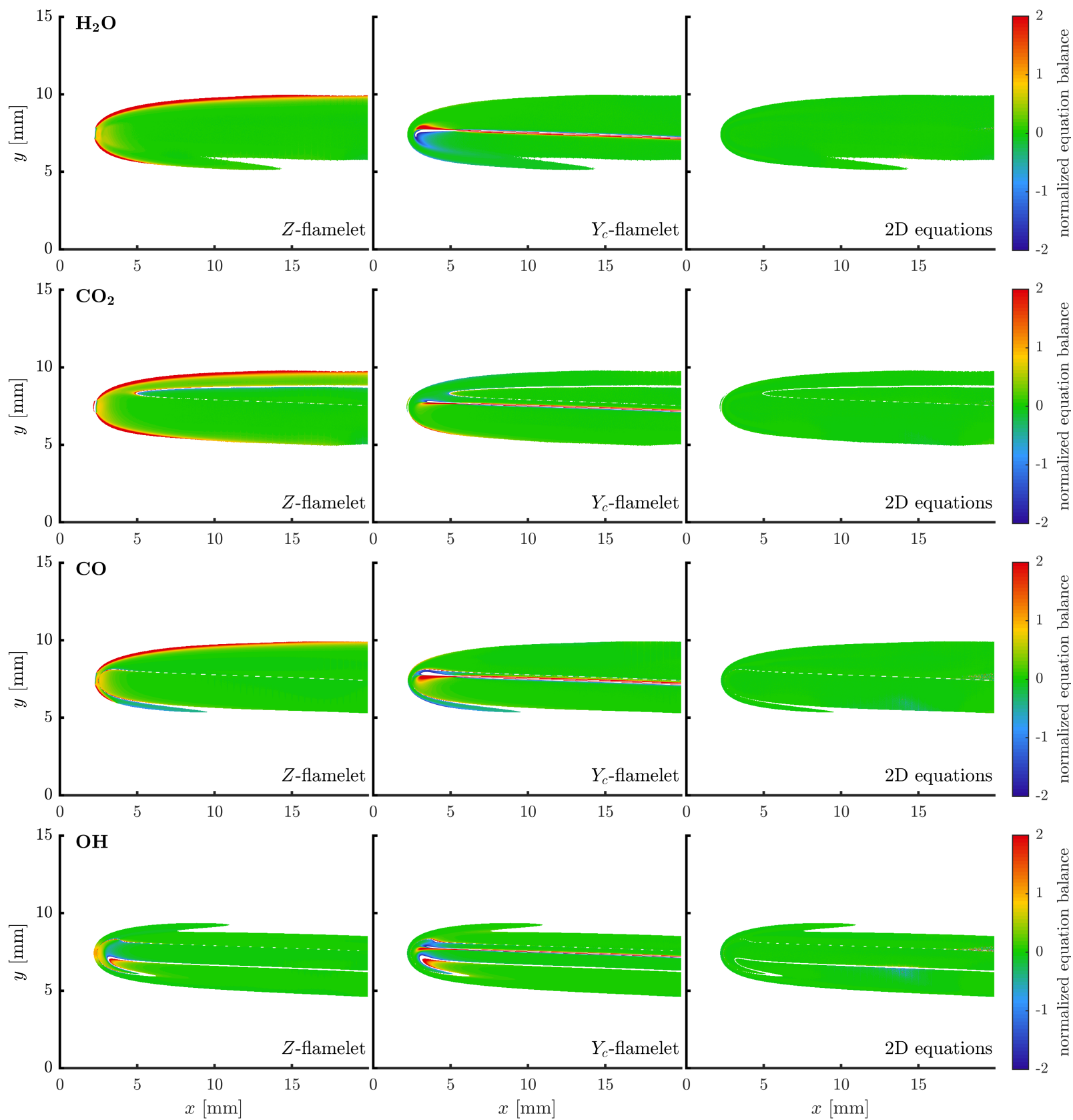

Figure 10: Normalized balances of composition space equations for the species $\mathrm{H}_{2} \mathrm{O}, \mathrm{CO}_{2}, \mathrm{CO}$ and OH. Left column: balance of the flamelet equations for non-premixed ( $Z$-)flamelets, Eq. (34). Middle: balance of the flamelet equations for premixed $\left(Y_{c^{-}}\right)$flamelets, Eq. (30). Right: balance of the 2D composition space equations, Eq. (27). All terms were post-processed from the detailed chemistry solution of the triple flame and then summed up to yield the respective equation balance. Then, the balance values were normalized with the local chemical source term. Points are shown for which the chemical source term of the respective species is larger than $0.1 \%$ of its maximum value in the computational domain. 
space equations avoiding cross-terms. Besides this, an extension of the composition space equations to incorporate differential diffusion and curvature effects would represent a valuable addition.

\section{Acknowledgments}

Financial support is kindly acknowledged from the Deutsche Forschungsgemeinschaft(DFG, German Research Foundation) - Projektnummer 325144795.

\section{References}

[1] E. Knudsen, H. Pitsch, Capabilities and limitations of multi-regime flamelet combustion models, Combust. Flame 159 (2012) 242-264.

[2] A. Masri, Partial premixing and stratification in turbulent flames, Proc. Combust. Inst. 35 (2015) $1115-1136$.

[3] S. Hartl, R. Van Winkle, D. Geyer, A. Dreizler, G. Magnotti, C. Hasse, R. Barlow, Assessing the relative importance of flame regimes in Raman/Rayleigh line measurements of turbulent lifted flames, Proceedings of the Combustion Institute 37 (2019) 2297-2305.

[4] D. Butz, S. Hartl, S. Popp, S. Walther, R. S. Barlow, C. Hasse, A. Dreizler, D. Geyer, Local flame structure analysis in turbulent $\mathrm{CH} 4 /$ air flames with multi-regime characteristics, Combust. Flame (2019).

[5] H. Yamashita, M. Shimada, T. Takeno, A numerical study on flame stability at the transition point of jet diffusion flames, Symp. (Int.) Combust. 26 (1996) 27-34.

[6] P. Domingo, L. Vervisch, K. Bray, Partially premixed flamelets in LES of nonpremixed turbulent combustion, Combust. Theor. Model. 6 (2002) 529-551.

[7] Y. Mizobuchi, S. Tachibana, J. Shinio, S. Ogawa, T. Takeno, A numerical analysis of the structure of a turbulent hydrogen jet lifted flame, Proc. Combust. Inst. 29 (2002) 2009-2015.

[8] P. Domingo, L. Vervisch, J. Reveillon, DNS analysis of partially premixed combustion in spray and gaseous turbulent flame-bases stabilized in hot air, Combust. Flame 140 (2005) 172-195.

[9] Y. Mizobuchi, J. Shinjo, S. Ogawa, T. Takeno, A numerical study on the formation of diffusion flame islands in a turbulent hydrogen jet lifted flame, Proc. Combust. Inst. 30 (2005) 611-619.

[10] B. Fiorina, O. Gicquel, L. Vervisch, S. Carpentier, N. Darabiha, Approximating the chemical structure of partially premixed and diffusion counterflow flames using FPI flamelet tabulation, Combust. Flame 140 (2005) 147-160.

[11] E. Knudsen, H. Pitsch, A general flamelet transformation useful for distinguishing between premixed and non-premixed modes of combustion, Combust. Flame 156 (2009) 678-696. 
[12] S. Hartl, D. Geyer, A. Dreizler, G. Magnotti, R. S. Barlow, C. Hasse, Regime identification from Raman/Rayleigh line measurements in partially premixed flames, Combust. Flame 189 (2018) 126 141.

[13] N. Peters, Laminar flamelet concepts in turbulent combustion, Symp. (Int.) Combust. 21 (1988) $1231-1250$.

[14] J. A. van Oijen, A. Donini, R. J. M. Bastiaans, J. H. M. ten Thije Boonkkamp, L. P. H. de Goey, State-of-the-art in premixed combustion modeling using flamelet generated manifolds, Prog. Energy Combust. Sci. 57 (2016) $30-74$.

[15] O. Gicquel, N. Darabiha, D. Thévenin, Laminar premixed hydrogen/air counterflow flame simulations using flame prolongation of ILDM with differential diffusion, Proc. Combust. Inst. 28 (2000) $1901-$ 1908.

[16] B. Fiorina, R. Baron, O. Gicquel, D. Thévenin, S. Carpentier, N. Darabiha, Modelling non-adiabatic partially premixed flames using flame-prolongation of ILDM, Combust. Theor. Model. 7 (2003) 449-470.

[17] R. Cònsul, A. Oliva, C. D. Pérez-Segarra, D. Carbonell, L. de Goey, Analysis of the flamelet concept in the numerical simulation of laminar partially premixed flames, Combust. Flame 153 (2008) 71-83.

[18] S. Popp, F. Hunger, S. Hartl, D. Messig, B. Coriton, J. H. Frank, F. Fuest, C. Hasse, LES flameletprogress variable modeling and measurements of a turbulent partially-premixed dimethyl ether jet flame, Combust. Flame 162 (2015) 3016-3029.

[19] W. Han, A. Scholtissek, C. Hasse, The role of tangential diffusion in evaluating the performance of flamelet models, Proc. Combust. Inst. 37 (2019) 1767-1774.

[20] X. Wen, Y. Luo, K. Luo, H. Jin, J. Fan, LES of pulverized coal combustion with a multi-regime flamelet model, Fuel 188 (2017) 661-671.

[21] P. Domingo, L. Vervisch, D. Veynante, Large-eddy simulation of a lifted methane jet flame in a vitiated coflow, Combust. Flame 152 (2008) $415-432$.

[22] P.-D. Nguyen, L. Vervisch, V. Subramanian, P. Domingo, Multidimensional flamelet-generated manifolds for partially premixed combustion, Combust. Flame 157 (2010) $43-61$.

[23] C. Hasse, N. Peters, A two mixture fraction flamelet model applied to split injections in a DI diesel engine, Proc. Combust. Inst. 30 (2005) 2755-2762.

[24] E. M. Doran, H. Pitsch, D. J. Cook, A priori testing of a two-dimensional unsteady flamelet model for three-feed combustion systems, Proc. Combust. Inst. 34 (2013) 1317-1324.

[25] D. J. Cook, H. Pitsch, G. Nentwig, Numerical investigation of unburnt hydrocarbon emissions in a homogeneous-charge late-injection diesel-fueled engine, SAE Technical Paper Series (2008).

[26] V. Mittal, D. J. Cook, H. Pitsch, An extended multi-regime flamelet model for IC engines, Combust. Flame 159 (2012) 2767-2776. 
[27] A. Kronenburg, Double conditioning of reactive scalar transport equations in turbulent nonpremixed flames, Phys. Fluids 16 (2004) 2640-2648.

[28] A. Scholtissek, P. Domingo, L. Vervisch, C. Hasse, A self-contained progress variable space solution method for thermochemical variables and flame speed in freely-propagating premixed flamelets, Proc. Combust. Inst. 37 (2018) 1529-1536.

[29] A. Scholtissek, P. Domingo, L. Vervisch, C. Hasse, A self-contained composition space solution method for strained and curved premixed flamelets, Combust. Flame 207 (2019) 342-355.

[30] R. W. Bilger, The structure of diffusion flames, Combust. Sci. Technol. 13 (1976) 155-170.

[31] M. Matalon, On flame stretch, Combust. Sci. Technol. 31 (1983) 169-181.

[32] A. Scholtissek, F. Dietzsch, M. Gauding, C. Hasse, In-situ tracking of mixture fraction gradient trajectories and unsteady flamelet analysis in turbulent non-premixed combustion, Combust. Flame 175 (2017) 243-258.

[33] C. D. Pierce, P. Moin, Progress-variable approach for large-eddy simulation of non-premixed turbulent combustion, J. Fluid Mech. 504 (2004) 73-97.

[34] G. Lodier, L. Vervisch, V. Moureau, P. Domingo, Composition-space premixed flamelet solution with differential diffusion for in situ flamelet-generated manifolds, Combust. Flame 158 (2011) 2009 - 2016.

[35] M. Ihme, Y. C. See, Prediction of autoignition in a lifted methane/air flame using an unsteady flamelet/progress variable model, Combust. Flame 157 (2010) 1850-1862.

[36] C. Hasse, N. Peters, Modelling of ignition mechanisms and pollutant formation in direct-injection diesel engines with multiple injections, Int. J. Engine Res. 6 (2005) 231-246.

[37] B. Savard, G. Blanquart, Effects of dissipation rate and diffusion rate of the progress variable on local fuel burning rate in premixed turbulent flames, Combust. Flame 180 (2017) 77-87.

[38] N. Peters, Laminar diffusion flamelet models in non-premixed turbulent combustion, Prog. Energy Combust. Sci. 10 (1984) 319-339.

[39] H. Pitsch, M. Chen, N. Peters, Unsteady flamelet modeling of turbulent hydrogen-air diffusion flames, Symp. (Int.) Combust. 27 (1998) 1057-1064.

[40] F. Dietzsch, A. Scholtissek, F. Hunger, C. Hasse, The impact of thermal diffusion on the structure of non-premixed flames, Combust. Flame 194 (2018) 352-362.

[41] A. Scholtissek, R. Pitz, C. Hasse, Flamelet budget and regime analysis for non-premixed tubular flames, Proc. Combust. Inst. (2016) 1349-1356.

[42] J. Boulanger, L. Vervisch, Diffusion edge-flame: approximation of the flame tip Damköhler number, Combust. Flame 130 (2002) 1-14.

[43] G. P. Smith, D. M. Golden, M. Frenklach, N. W. Moriarty, B. Eiteneer, M. Goldenberg, C. T. Bowman, R. K. Hanson, S. Song, W. C. G. Jr., V. V. Lissianski, Z. Qin, Gri-mech 3.0, online, 1999. URL: 
http://combustion.berkeley.edu/gri-mech/.

[44] Y. Wu, C. Cao, T. Ye, Q. Lin, A new multi-dimensional flamelet generated manifolds approach for approximating partially premixed flame structure, J. Therm. Sci. Technol. 10 (2015) JTST0017JTST0017. 